\title{
Information system for interpreting the results of pharmacogenetic testing of patients with mental and behavioral disorders
}

\author{
A. Martynenka ${ }^{1}$, T. Dakukina ${ }^{1}$, T. Golubeva ${ }^{1}$, A. Gilep $^{2}$, I. Haidukevich ${ }^{2}$ \\ ${ }^{1}$ Republican scientific-practical center of mental health, Department of mental and behavioral disorders, \\ Minsk, Belarus. \\ 2 Institute of Bioorganic Chemistry, department of molecular biotechnologies, Minsk, Belarus.
}

\section{Objectives:}

Automation of the process of formation of the conclusion on the basis of the identified genotype of the patient for timely treatment, taking into account the individual characteristics of the patient.

\section{Background:}

Depending on the combination of allelic variants of the genes, encoding drug metabolizing enzymes, there is a choice of the clinical interpretation of the results of pharmacogenetic testing of patients for each drug.

\section{Materials and Methods:}

- The automated system includes list of more than 40 drugs certified in Republic of Belarus for treatment mental and behavioral disorders.

- Results of pharmacogenetic testing include a list of allelic variants of CYP 450 genes: CYP2D6 * 4, CYP1A2 * 1F, CYP2C9 *2, CYP2C9 * 3, CYP2C19* 2, CYP2C19 * 17 and C3435T polymorphism of MDR1 gene. Genomic DNA was extracted from patient's biological material (saliva) and genotyped utilized PCR-RFLP method.

- Pharmacogenetic information were obtained from 1100 patients with mental and behavioral disorders, which were treated with utilization of pharmacogenetic testing.

- The therapeutical effect of personalized treatment was estimated with morphological, clinic-functional, pathopsychological and statistical methods.

\section{Results and Conclusions:}

Developed information system for interpreting the results of pharmacogenetic testing of patients with mental and behavioral disorders is based on unique algorithm and allows to obtain medical report automatically. Medical report includes two parts. The first part represents a list of identified allelic variants of pharmacogenes, that affect the drug metabolism, in a particular patient, with description of the type of the effect. In the second part of the report, there is an interpretation of the results of pharmacogenetic testing, indicating the characteristics of the metabolism of each drug with recommendations for dose adjustment and drug of choice for a given patient. Utilization of developed information system in routine medical practice allow psychiatrists without deep knowledges in genetics and biochemistry of psychiatric drug metabolism to provide the effective drug treatment with minimized risk of ADRs.

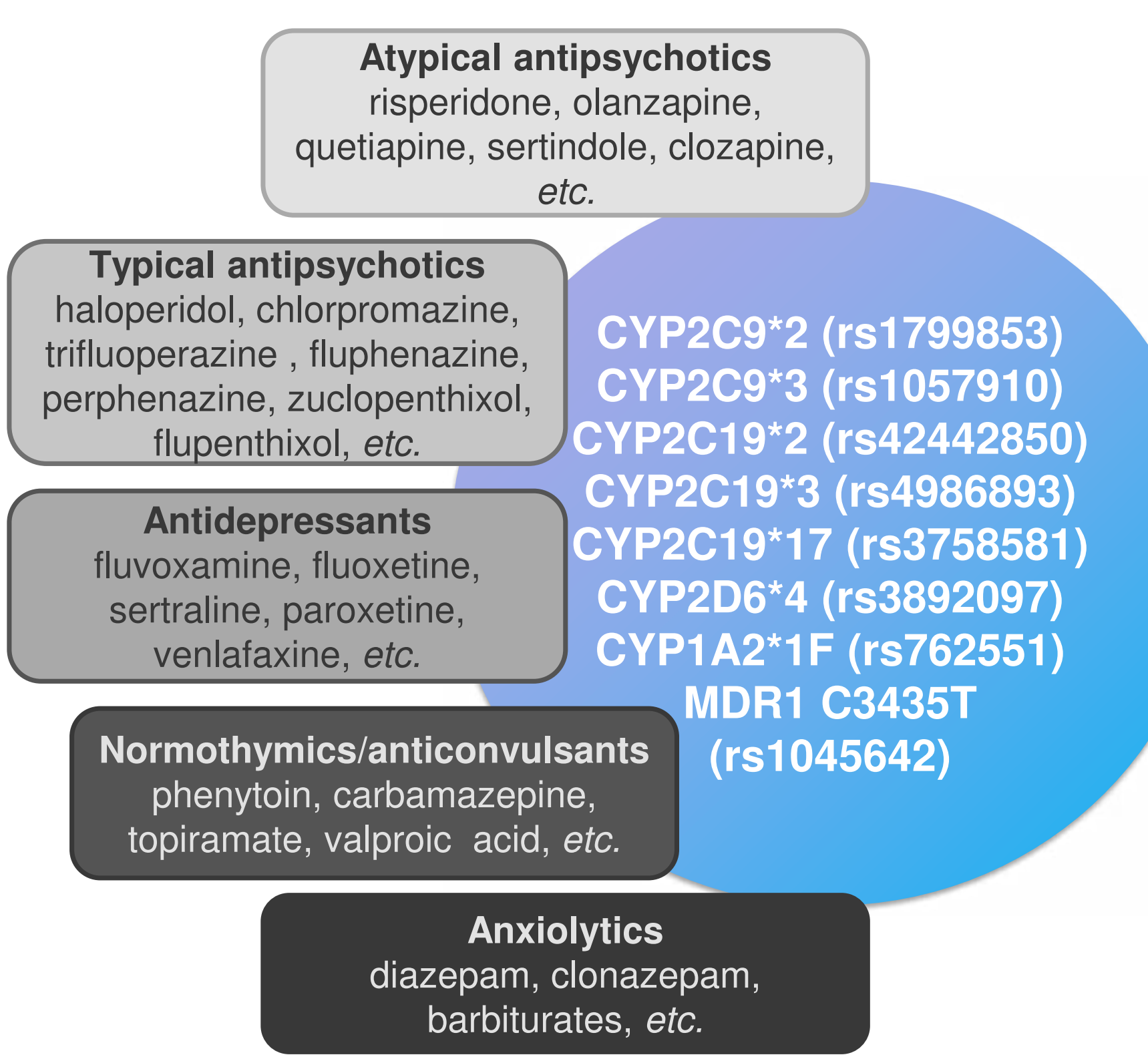

Copyright $\odot 2019$

A. Martynenka, T. Dakukina*, T. Golubeva, A. Gilep, I. Haidukevich *email: science@mentalhealth.by 